OPEN ACCESS

Edited by:

Andrea Pieroni,

University of Gastronomic

Sciences, Italy

Reviewed by:

Alfred Maroyi,

University of Fort Hare, South Africa

Barbora Duži,

Institute of Geonics (ASCR), Czechia

Joan Vallès,

University of Barcelona, Spain

*Correspondence:

Leonie K. Fischer

leonie.fischer@ilpoe.uni-stuttgart.de

Specialty section

This article was submitted to

Urban Agriculture,

a section of the journal

Frontiers in Sustainable Food Systems

Received: 22 December 2021

Accepted: 21 January 2022

Published: 02 March 2022

Citation:

Cerda C, Guenat S, Egerer $M$ and

Fischer LK (2022) Home Food

Gardening: Benefits and Barriers

During the COVID-19 Pandemic in

Santiago, Chile.

Front. Sustain. Food Syst. 6:841386.

doi: 10.3389/fsufs. 2022.841386

\section{Home Food Gardening: Benefits and Barriers During the COVID-19 Pandemic in Santiago, Chile}

\author{
Constanza Cerda ${ }^{1}$, Solène Guenat ${ }^{1}$, Monika Egerer ${ }^{2}$ and Leonie K. Fischer ${ }^{1 *}$ \\ ${ }^{1}$ Institute of Landscape Planning and Ecology, University of Stuttgart, Stuttgart, Germany, ${ }^{2}$ Department of Life Science \\ Systems, TUM School of Life Sciences, Technical University of Munich, Freising, Germany
}

The recent COVID-19 pandemic has changed the way people live in an unprecedented way. This includes severe impacts on people's health and wellbeing such as stress, reduced physical activity and loneliness due to confinement. In parallel, people had to find ways to secure their food, with fresh food especially scarce in some regions due to lockdowns and restricted flow of goods. As in previous massive crises, the practice of home food gardening seemed to have increased during this pandemic. Yet we largely do not know which parts of society took to home food gardening, in which urban setting home food gardening was practiced, and to which extent this practice contributed to people's daily livelihoods during the pandemic. In this case study we explore (a) who practices home food gardening, (b) the garden characteristics, and (c) the main perceived benefits and barriers bound to home food gardening during the pandemic. We set up an online questionnaire targeted at the population of Santiago de Chile, for which we received 305 responses. Our results clearly show that the possibility of being in contact with nature and feeling less stress through practicing home food gardening were the most significant perceived benefits, while lack of or inadequate space to garden and limited knowledge on home food gardening were the main barriers. These findings highlight for the specific context of a metropolitan region in South America that access to gardens and green space could contribute to a just urban society. Likewise, the practice of home food gardening can be a relevant tool to cope with the adverse consequences of the pandemic by informing public initiatives to promote healthier lifestyles during this and future crises contexts and also in the awaited "ordinary" times.

Keywords: COVID-19 (SARS-CoV-2) pandemic, crisis gardening, gardening perception, public health, socialecological systems, spatial garden characteristics, urban vegetable farming, urban agriculture practices

\section{INTRODUCTION}

For centuries, home food gardens have been an essential component of families' self-provisioning practices all over the world (Galhena et al., 2013; Vávra et al., 2018). Home food gardens have evolved together with the way societies have urbanized (Ferreira et al., 2018). During social, economic, and environmental crises-times of intense difficulty, trouble, or dangerhome food gardens have risen in importance (Mullins et al., 2021), with some home food 
gardens maintained by households specifically in response to collective crises (Schupp and Sharp, 2012; Cepić and TomićevićDubljević, 2017; Katz, 2020; Figure 1).

The current COVID-19 pandemic, declared on March 11, 2020 (World Health Organization, 2020) and relating to the highly contagious coronavirus (SARS-CoV-2), is a contemporary crisis facing society. In many countries, initial reactions to hinder the spread of the coronavirus included the closure of international borders and the implementation of curfews and social distancing to restrict and control social contact. The global spread of the virus called for almost half of the world's population in more than 90 countries to stay at home (Sandorf, 2020). These global efforts to reduce the spread of the disease have had unintended impacts on people's health and wellbeing. Isolation from others including family members, restricted time outdoors, and the reorganization of life at home-work, studies, and/or

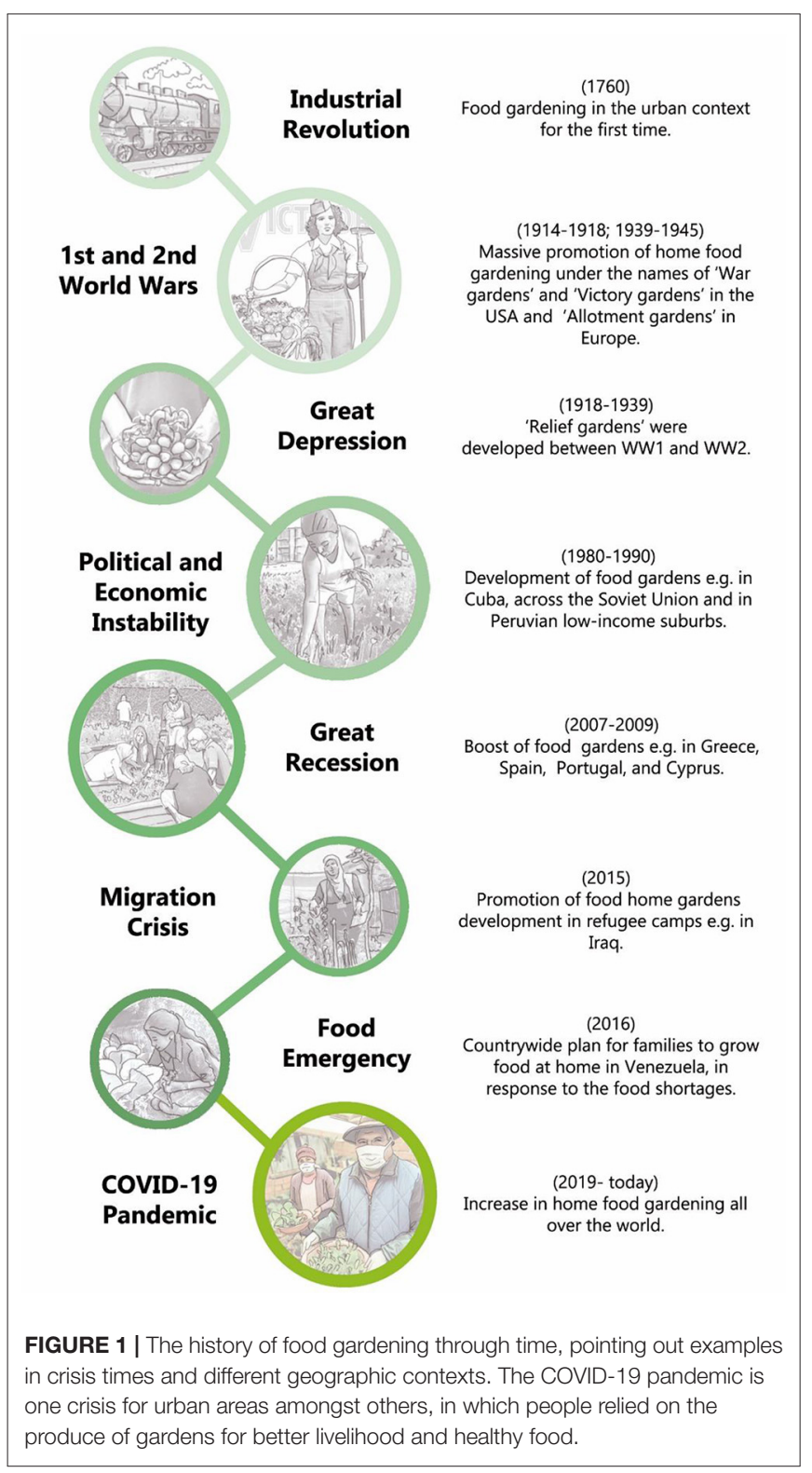

childcare-have strongly affected people's lifestyles (Lades et al., 2020; Garre-Olmo et al., 2021). Recent studies revealed the severe consequences of this confinement on human mental and physical health, with increased levels of e.g., stress, loneliness, and depression (Lades et al., 2020; Buckner et al., 2021; Kasar and Karaman, 2021; Pouso et al., 2021), as well as a generalized increase in sedentary lifestyles (Cheval et al., 2020). In parallel, alterations in trade networks due to international and local restrictions strongly affected the food systems (Ma et al., 2021; Rivera-Ferre et al., 2021). This amplified the risk of severe food insecurity and added, by the end of 2020, 265 million people to the already 821 million suffering from hunger worldwide (United Nations, 2020).

Home food gardens have heightened in relevance during this time due to their accessibility in time of movement restriction and their capacity to contribute to fresh food provision (Sofo and Sofo, 2020; Corley et al., 2021; Ma et al., 2021), aid in stress relief (Sunga and Advincula, 2021), promote physical activity (Machida, 2019), and contribute to strengthening social relations (Katz, 2020; Darly et al., 2021). This translated into a surge in home food gardening in cities around the world during the pandemic (Chenarides et al., 2020; Montefrio, 2020; Mullins et al., 2021), highlighting how urban agriculture can serve urban populations in times of crisis. Nevertheless, there is little insight on what influenced or motivated people to garden during this time, and specifically, whether gardening experience and associated knowledge and/or equipment were motivators and/or hindrances for home food gardening uptake during the pandemic (Chenarides et al., 2020; Katz, 2020). Furthermore, previous research on home food gardens in times of crisis mainly focused on food security issues (Galhena et al., 2013; Herrmann, 2015; Montefrio, 2020). Research focus was also largely on community gardens, at the expense of home food gardens within the private realm, despite the critical role of the private sphere in the COVID-19 context (Ma et al., 2021; Mullins et al., 2021; Sunga and Advincula, 2021). This highlights the relevance of examining the multifunctionality and changing role of urban agriculture in general and home food gardens in particular.

The city of Santiago de Chile presents an opportunity to understand the benefits and barriers of home food gardening in an urban context during the COVID-19 pandemic. The large and densely populated city was highly affected by the pandemic, with a city-wide lockdown of about 200 days. The cultivation of food within Santiago has increased in popularity in recent years, especially due to the creation of community gardens by local communities such as NGOs, artists, neighborhoods, and university groups (Contesse et al., 2018). Previous research has shown the public character of community gardens (Casanova, 2016), the contribution of gardens to biodiversity (Herrera, 2020), and policy approaches to promote community gardens (Contesse et al., 2018). Yet, home food gardens have been largely overlooked in research, despite amounting to $33 \%$ of food gardens in Santiago (Casanova, 2016). This is reflected in public initiatives implemented to support food gardening activities focusing exclusively on community gardens (Contesse et al., 2018), and calls for research to understand home food gardening as a relevant practice in Santiago. 
We aim to describe the socio-demographic characteristics of home food gardeners and the garden characteristics during a period of the COVID-19 pandemic. We examine the multiple barriers people faced while growing food at home and the benefits they gained from home food gardening during a period of the COVID-19 pandemic. We explore whether there are differences in perceptions of barriers and benefits, as well as in socio-demographic characteristics, between people who took up home food gardening before the pandemic and those who started during the pandemic. Finally, we describe the other socio-demographic characteristics of home food gardeners and the garden characteristics. This understanding aims to inform public initiatives during this and future crises contexts and in the awaited "ordinary" times. We asked the following research questions:

(1) Do the socio-demographic background of gardeners and the physical characteristics of their gardens differ according to the time of gardening uptake?

(2) What are the main perceived barriers on home food gardening uptake and the main benefits on health and wellbeing from home food gardening practices during the COVID19 pandemic?

(3) How do barriers and benefits differ between those who took up home food gardening pre- or during the pandemic?

\section{METHODS}

\section{Study Area}

The study was conducted in the city and province of Santiago, the capital of Chile. It is located in the central valley between the Coastal and Andes mountains at 567 m.a.s.l., covering an area of $2,030.3 \mathrm{~km}^{2}$. As the most populated city in Chile, with 5.250 .565 inhabitants, it is characterized by a high level of urbanization (INE, 2017). The built area corresponds to $60 \%$ of the total area, growing by an average of 1,273 ha per year (Inostroza et al., 2013). Santiago has 32 districts, each one with its independent municipality (Contesse et al., 2018). Districts differ in regards to their economic means, educational and healthcare capability, and the way they deal with inequalities. Specifically, there tends to be an unequal access to greenspaces, with public greenspaces being distributed inversely proportionally to the income of the districts. $52.6 \%$ of Santiago's greenspaces are private gardens, as opposed to $13 \%$ being publicly accessible green areas, with the others consisting of street trees (Espinoza, 2018). The most common residential typologies in Santiago can be organized into four main types, that are: (a) high-density residential buildings, usually with a small balcony, and few common greenspaces; (b) lowdensity residential buildings, organized around a common green area or park; (c) family houses with a front yard or backyard, most common in mid and high-income districts; and (d) highdensity family houses with almost no gardens, usually related to low-income districts.

\section{The Pandemic Context}

The first case of a COVID-19 infection in Chile was confirmed on March 3, 2020. As the virus spread rapidly, the city of
Santiago suffered several massive lockdowns in all its districts since May 2020. Between May 2020 and June 2021, the most severe restrictions did not allow people to leave their homes except if they had a special permit, even if it was for getting groceries or going for a walk. Access to public greenspaces, such as city-scale parks, neighborhood green areas, and community gardens, was also restricted. People were thus highly limited in their possibility to visit greenspaces, reducing their possibilities of having contact with nature to their private space.

\section{Survey Development}

We developed a questionnaire survey for people who practiced home food gardening during the COVID-19 pandemic. The questionnaire was developed on the basis of four semi openended interviews with people engaged in home food gardening during the pandemic and a literature review. The questionnaire was initially designed in English, then translated and distributed in Spanish, considering local language features. The online questionnaire was set up online in the SoGo Survey platform, with a total of 20 questions designed to be answered only by people who practiced home food gardening during the COVID19 pandemic within the Province of Santiago de Chile. The survey included a statement regarding the consent to participate (being older than 18), and regarding data privacy, indicating that all responses were treated anonymously (full questionnaire in Supplementary Table 1).

The survey included multiple items on the socio-demographic background of respondents and their garden settings, and the perceived barriers and benefits from home food gardening (Table 1). To answer the first research question, we asked in the survey about (a) gardening experience (first experience in home food gardening, time of their gardening uptake; the latter was used to differentiate between people starting gardening preor during the pandemic throughout the analyses of the data set), (b) respondents' pre-pandemic relationship with public greenspaces, (c) socio-demographic characteristics, including gender, age, household size and social priority (based on the district), (d) where people gardened (place, location), and (e) the physical characteristics of these home food gardens (accessibility, size). To answer the second and third research questions, two items were included on these themes, namely: (f) the perceived barriers, leaving respondents five categories to choose from, combined with an open entry in which respondents were able to freely write up to 100 characters. This information was made available to complement the data obtained concerning benefits and barriers from a more qualitative perspective, and ( $g$ ) the perceived benefits, showing respondents six categories, in which they rated each potential benefit on a 5-points Likert scale. Finally, (h) a question asking respondents if they plan to continue gardening in the future was included. The English version of the questionnaire is included in Supplementary Table 1.

\section{Survey Distribution}

The questionnaire was distributed online between March 22nd and April 25th, 2021. During this time span, all districts in Santiago were under lockdown due to the second wave of COVID-19 cases. Consequently, face-to-face distribution of the 
TABLE 1 | Themes and variables with answer options derived from the online survey.

\begin{tabular}{|c|c|c|}
\hline Survey questions subject & Variables & Answer options \\
\hline \multirow[t]{2}{*}{ (a) Gardening experience } & First experience in home food gardening & Childhood; adulthood \\
\hline & Time of gardening uptake & Pre-pandemic; during the pandemic \\
\hline $\begin{array}{l}\text { (b) Pre-pandemic relationship with public } \\
\text { greenspaces }\end{array}$ & Frequency of visiting public greenspaces & Several times a week; once a week; less than once a week; never \\
\hline \multirow[t]{2}{*}{ (c) Socio-demographic characteristics } & Gender & Male; female \\
\hline & District & Open-ended. Used to extract the development level. \\
\hline \multirow[t]{2}{*}{ (d) Where people garden } & Place & House; apartment; other (describe) \\
\hline & Location & $\begin{array}{l}\text { Front or backyard; balcony; other (describe). Possibility to select } \\
\text { various options }\end{array}$ \\
\hline (e) Physical characteristics of gardens & Accessibility & Private; shared \\
\hline \multirow[t]{2}{*}{ (g) Perceived benefits } & $\begin{array}{l}\text { Social interaction } \\
\text { Feeling less stress } \\
\text { Contact with nature } \\
\text { Contribution to diet } \\
\text { Contribution to income } \\
\text { Being occupied }\end{array}$ & $\begin{array}{l}\text { 5-points Likert scale for each benefit, ranging from "totally agree" } \\
\text { to "totally disagree" }\end{array}$ \\
\hline & Barriers and benefits & Open entry \\
\hline
\end{tabular}

questionnaire, necessary to reach people with limited internet access, was not possible. At the beginning of February 2021, a specialized Instagram account [@huertas_en_pandemia (food gardens during the pandemic)] was created to generate a net of people related to home food gardening in Santiago. The distribution of the survey was developed through snowball sampling. It was initially sent through "direct" messages to the 360 followers of the Instagram account, with the recipients being encouraged to share the link between their contacts and social networks. The survey link was permanently available on the Instagram account profile and was published as a "story" as well.

\section{Data Validation and Editing}

Out of 430 responses, 125 were left out of the analyses because respondents did not declare living in Santiago city. The remaining 305 responses serve as the database for this study. This data sample was standardized; for example, responses to open-ended questions that fitted pre-defined answer categories were included in the respective pre-defined category. Districts were classified depending on their development levels based on the Social Priority Index for Districts, 2019, which takes into consideration income, access to health services and education (Gajardo, 2019). The original five categories: high, mid-high, mid-low, low and no social priority were combined into three categories: low development level (high, mid-high, and midlow social priorities), medium development levels (low social priority), and high development levels (no social priority). We excluded from the analysis on the related themes any respondent who did not answer regarding gender $(n=3)$, the different benefits ( $n=27$ for socializing, $n=8$ for more contact with nature, $n=4$ for contribution to the diet, $n=12$ for contribution to the income, and $n=2$ for being occupied) and, for garden place, we excluded all data $(n=7)$ that were neither a house nor an apartment.

\section{Statistical Analyses}

We analyzed whether there were significant differences for all variables according to whether respondents took up gardening before or during the pandemic. We used Pearson's Chi-square test to analyze all nominal variables, namely whether respondents identified each of the six barriers (lack of or inadequate space, lack of time, lack of access to resources, limited knowledge, experience of disagreement with others, and other barriers), when was their first experience in home food gardening, with which gender they identified, where they garden in terms of both their home environment and the location of their garden within the home, and whether their garden was private or shared. We used Student's $t$-tests to analyze all ordinal variables, namely the number of barriers they perceived, to which level they agreed 
that urban gardening provided each of the six benefits, their prepandemic frequency of greenspace visits, their socio-economic background such as age group, household size, within which level of social priority their neighborhood was located, and the size of their garden. To describe our sample, socio-economic variables were presented alongside data from the 2017 census (INE, 2017), except for household size, for which data were not available. All analysis were carried out with R, v.4.1.2 (R Core Team).

\section{RESULTS}

\section{Socio-Demographic Background of Gardeners}

In our survey, almost half of the respondents (47.87\%) started home food gardening during the recent COVID-19 pandemic (Supplementary Table 2). Whether they took up gardening preor during the pandemic, urban food gardeners came mostly from districts of high (46\%) and medium (39\%) development level. This is opposed to Santiago's population, of which $50 \%$ live in districts with low development level (Figure 2A; Supplementary Table 2). While no household size data were available for Santiago, we found that large households of more than four people were those most likely to garden $(51.5 \%$; Figure 2B). Women were the main participants in home food gardening, however their proportion within our respondents was on par with Santiago's gender distribution (Figure 2C). Urban gardeners were in general younger than the overall population, with the residents over 65 years old barely represented (1\%) and none taking up gardening after the start of the pandemic (Figure 2D). Yet, gardeners with pre- and during pandemic uptake did not significantly differ in terms of their sociodemographics (Supplementary Table 3).

Respondents mainly had their first experience of home food gardening in their adulthood (62\%, Figure 3B). They mostly had a close relationship with public greenspaces before the pandemic, with more than half of the respondents visiting public greenspaces at least once a week, as opposed to only 3.9\% who never visited public greenspaces (Figure 3C). Both the time of the first gardening experience and the frequency of greenspace visits were consistent across the respondents, with no significant difference according to their time of gardening uptake (Supplementary Table 3). Almost all respondents $(99.3 \%)$ planned on continuing to garden in the future (Figure 3D; Supplementary Table 3). There was no significant difference in the socio-demographic characteristics of the respondents depending on the time of gardening uptake (Figure 3A; Supplementary Table 3).

Almost all food gardens were located on private land (91.8\%), with only $8.2 \%$ having a shared access (Figure 4B; Supplementary Table 2). The majority of respondents lived and gardened in or around houses (70.8\%) as opposed to those living or gardening in or around apartments (26.9\%; Figure 4C). Front or backyards were the most common place to locate food gardens (55.4\%), with balconies being the second most important place for including a home food garden $(21.3 \%)$. Additionally, about one out of six participants (17.4\%) declared having several gardens in various locations, including both front

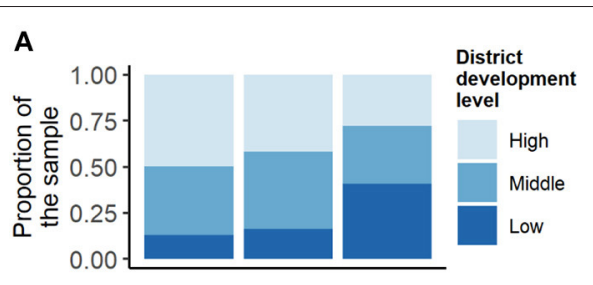

B

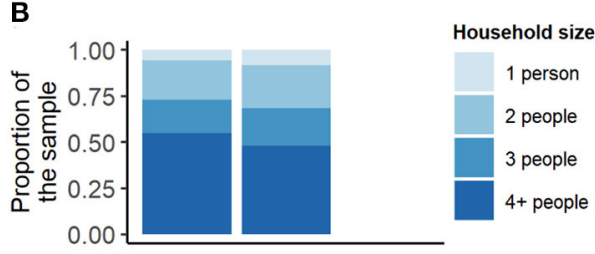

C
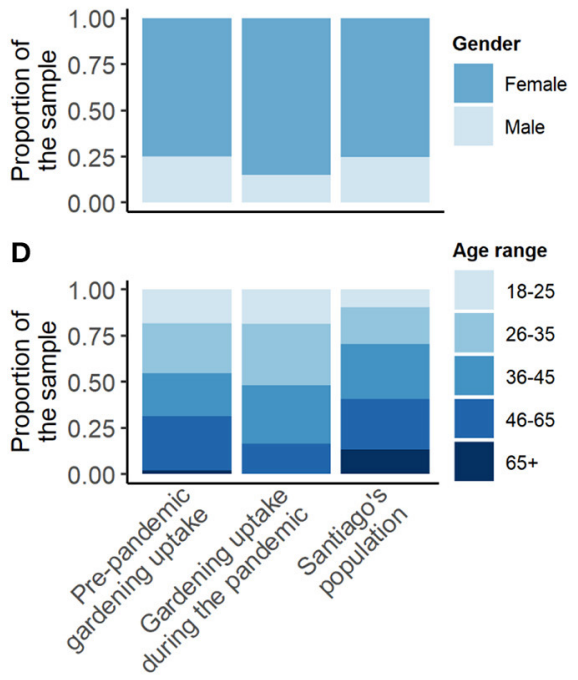

FIGURE 2 | Socio-demographic characteristics of the respondents according to whether they took up gardening pre- or during the pandemic, and in comparison to Santiago's population. (A) District development level; (B) Household size, no data were available for Santiago's population; (C) Gender; (D) age range, with the categories for Santiago's population being slightly different, namely 20-24, 25-34, 35-44, 45-64, 65+. There were no significant differences in the repartition of the population according to their time of gardening uptake.

and backyards and balconies, or other locations (Figure 4D). Less common locations included roofs, walls, window sills, sidewalks, road medium strips or parking spaces. Most of the respondents considered their gardens to be either small (63.9\%) or medium-sized (56.7\%), with only $9.2 \%$ of respondents perceiving their garden as large (Figure 4E). There was no significant difference in the physical characteristics of home food gardens depending on the time of gardening uptake (Figure 4A; Supplementary Table 3).

\section{Perceived Barriers}

Within our sample, each respondent determined an average of two barriers $(\min =1, \max =6)$, regardless of when they took up gardening (Figure 5A). In addition to the five barriers presented in the survey, respondents identified eight additional, 


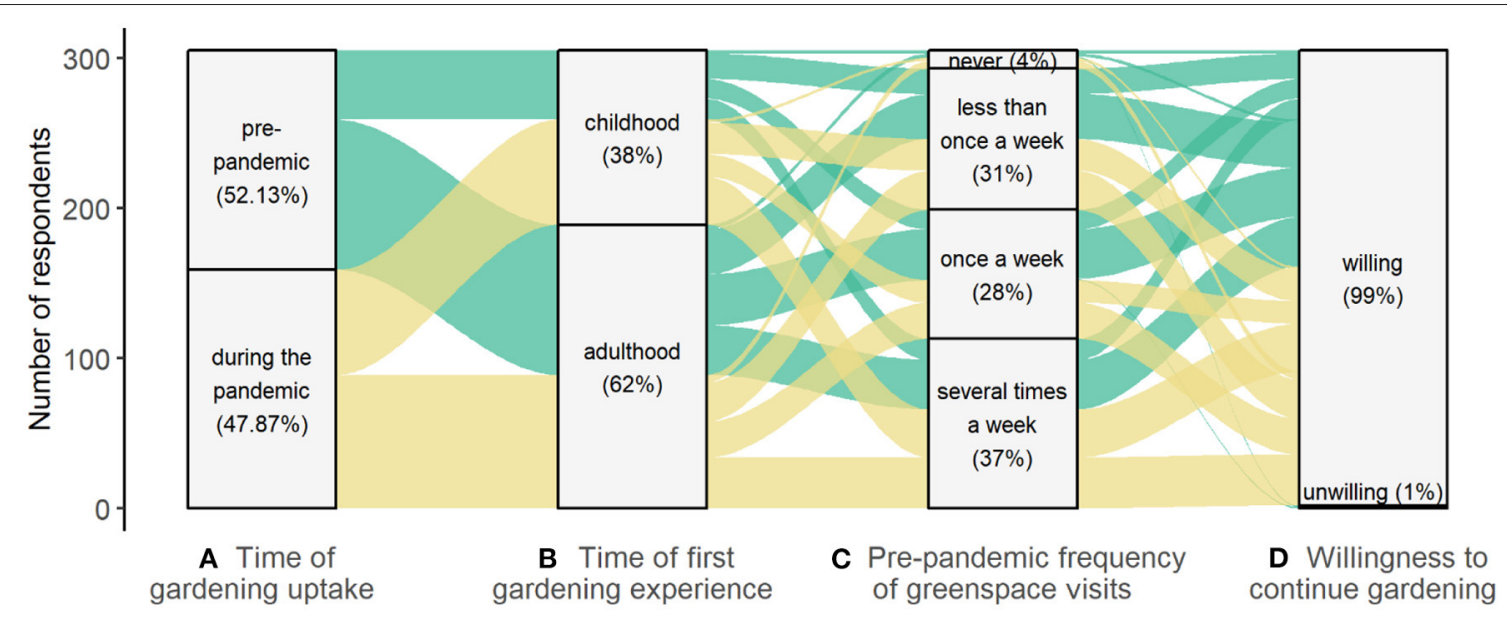

FIGURE 3 | Respondents' relationship with time of gardening uptake (A), time of first gardening experience (B), pre-pandemic frequency of greenspace visits (C), and (D) willingness to continue gardening after the pandemic. Colored lines indicate the proportion of respondents who took up gardening pre-pandemic (yellow) or during the pandemic (green). There were no significant differences according to the time of gardening uptake in relation to time of first gardening experience or pre-pandemic frequency of greenspace visits; for details see Supplementary Table 3.

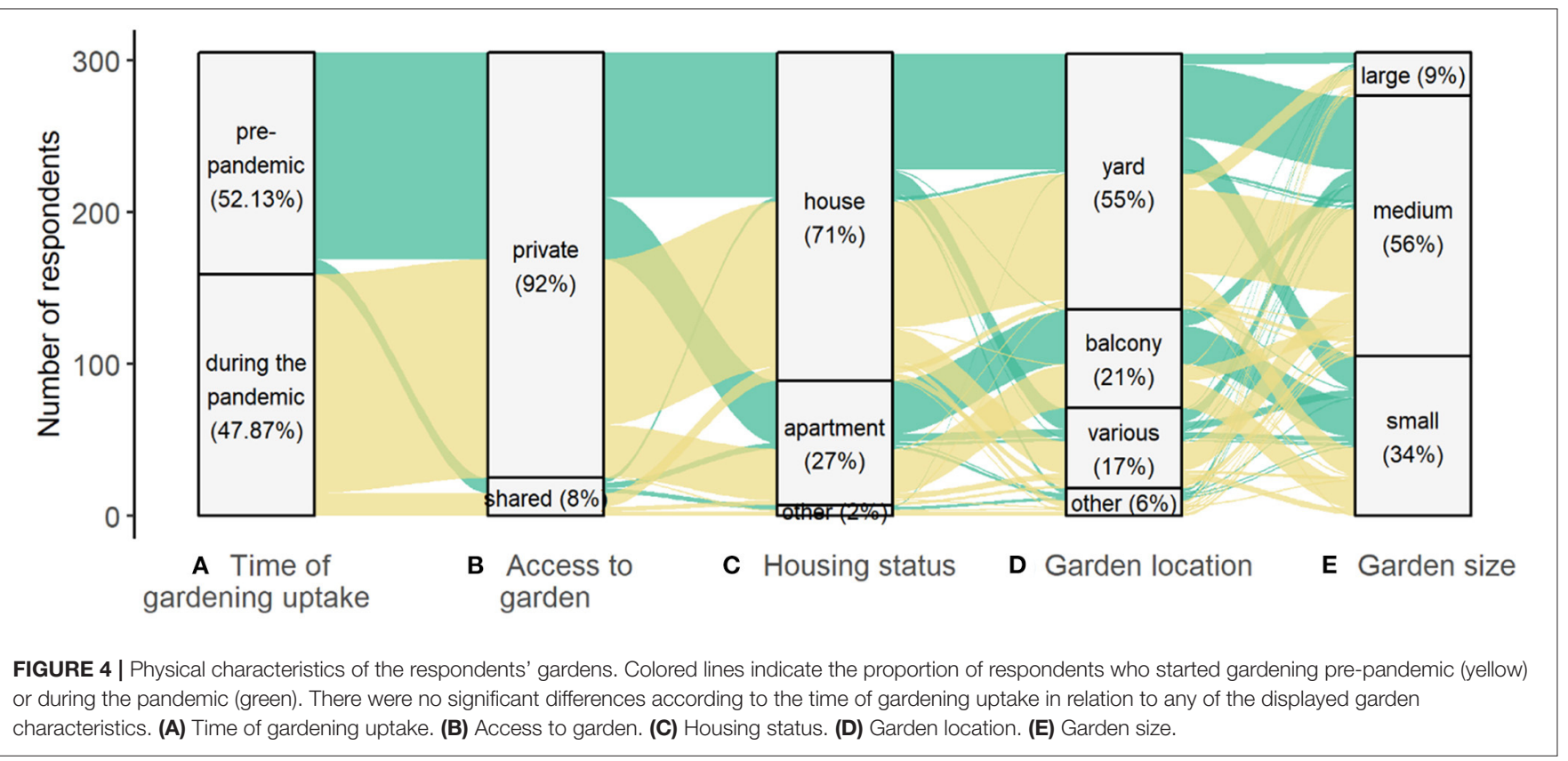

different barriers ranging from plague control (6.2\%), having little sun (2.3\%), not having enough access to the community (1\%), unwanted animals $(0.3 \%)$, and lack of personal motivation $(0.3 \%)$, amongst others. Overall, the most recognized barrier was the lack of space for gardening, with $49.8 \%$ of the respondents agreeing that it was an issue (Figure 5B), illustrated by answers to open-ended questions such as "Shared spaces in condominiums are sometimes not respected by neighbors, there is little space." The second most mentioned barrier was the limited knowledge (40\%), with respondents stating how "I have had to look up a lot of information on how to take care of plants and eliminate pests or fungi," followed by the lack of time (31.1\%). Some of the respondents however highlighted that they lacked time for gardening, and that the pandemic provided them with more time to enhance the practice they had started before. This was backed up for instance by stating how "this is my second year with food gardening and during the pandemic, I have been able to have a little more time and it is appreciated" or "by being more at home you can see in more detail the growth of the plants." Lack of resources, the third most mentioned barrier, was also mentioned with details of how resources lacking included water, soil, fertilizers and organic substances against pests, and how both "the current economic conditions reduce the possibility of obtaining better resources" and "it is ideal that people know how to navigate well on the Internet to get stuff, because of closed stores." 


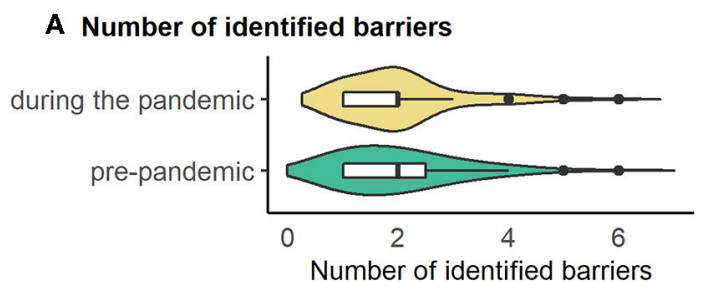

B Identified barriers

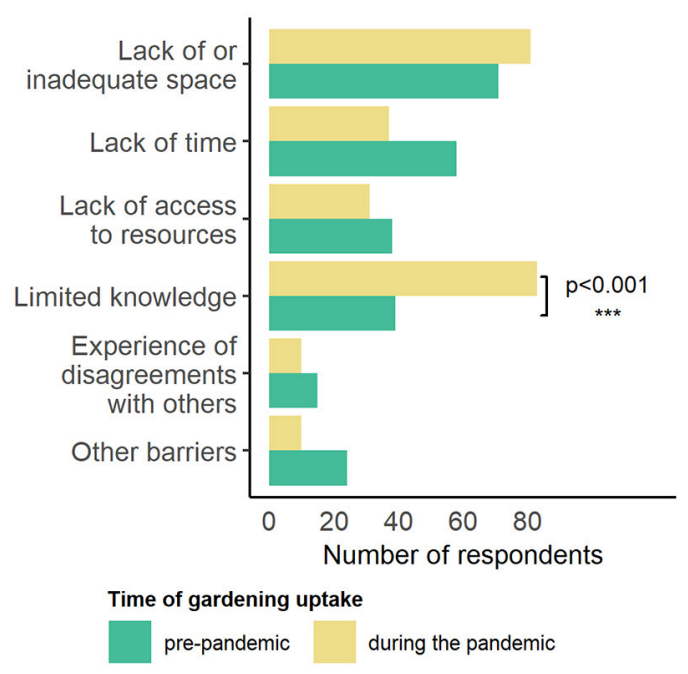

FIGURE 5 | (A) Number and (B) type of barriers identified by the respondents. Significance level indicated by ${ }^{\star \star \star} p<0.001$. For details on the test statistics, see Supplementary Table 3.

Limited knowledge of gardening practices was particularly problematic for those who took up gardening during the pandemic, as opposed to those who started before $\left(\chi^{2}=\right.$ $33.129, \mathrm{df}=1$, Bonferroni-adjusted $p$-value $<0.001$; Figure 5B; Supplementary Table 3 ). Time of gardening uptake had no influence on whether any of the other barriers were perceived as an impediment to home gardening (Figure 5B).

\section{Perceived Benefits}

Increased contact with nature was the benefit identified as important by most respondents, with $94 \%$ of the 305 respondents mentioning they either agreed or totally agreed with it (Figure 6). This was also described widely in open-ended questions, with answers such as "Personally, the garden has opened the way to ecological awareness and organic waste management," "I have become more conscious about caring for the environment. Being interested in composting," or "[Food gardening] has connected me with the origin of food, its times and cycles in nature." Most respondents also agreed that gardening helped them feel less stress $(84.9 \%)$, to the point that some stated how "[gardening] has been very therapeutic for me, my partner and my children," "cultivation in this pandemic has brought happiness and tranquility to my life. I am happy!!!" or "[home food gardening] got me out of depression." Gardening also contributed to keeping respondents occupied (71.5\%), becoming "an excellent activity and entertainment for the whole family. Especially for the children," improved their diet either in quantity or in quality $(68.5 \%)$, sometimes providing a life-line as when respondents stated that home food gardening "gives security that if you are not allowed to go out you have food at home" or "turned from being a hobby to a necessity." Though not a direct focus of the study, some respondents described the crops they were growing, which included at least chards, tomatoes, fruits and herbs, and how having access to some of those crops would not have been possible without gardening: "It allowed me to have herbs to which I wouldn't have had access during the pandemic otherwise as they are not sold close by." Gardening provided them with an opportunity to socialize (65.9\%), sometimes "allowing [them] to feel part of a community of people with the same interests and ideas." However, respondents were split as to whether gardening positively contributed to their income, with $39 \%$ of respondents mentioning it did, for instance by "allow[ing them] to reinvent [them] selves as a family (through a related small and mediumsized enterprise) and to eat healthier," while $32.8 \%$ of respondents stated it didn't and 22.9\% who weren't sure. Perceptions of the benefits were constant throughout our respondents, with no significant differences linked to the time of gardening uptake (Figure 6).

\section{DISCUSSION}

A renaissance in home food gardening is not new during crises. Food production at home is a common practice during economic crises, political upheaval, and natural disasters (Schupp and Sharp, 2012; Cepić and Tomićević-Dubljević, 2017; Shimpo et al., 2019; Mullins et al., 2021). Our study adds to the existing knowledge of drivers of home food gardening and how it is valued for multiple benefits, specifically within the COVID-19 pandemic. We uncover the barriers to home food gardening that could be addressed by urban planning to maintain this fruitful resource of benefits to wellbeing and physical health, especially considering the need to increase access to spaces used for food gardening across districts of lower development level. In our study in Santiago de Chile, people started gardening during the pandemic even if they did not have previous experience with home food gardening. Despite the barriers that affected them, a wide range of benefits such as allowing contact with nature, reducing stress, and socializing were particularly relevant. These results inform the practice of home food gardening during the pandemic, and how to support this activity in the future during and potentially beyond crises.

\section{Home Food Gardeners and Their Gardens}

Our results suggest that the COVID-19 pandemic crisis motivated the uptake of home food gardening among city dwellers in Santiago de Chile. Although home gardening is often considered a solitary activity, we showed that gardening was more likely to happen when people live in large households. Gardeners' household size was higher in Santiago than in the USA (Chenarides et al., 2020). However, gardeners from Santiago tend to live in smaller households than most of the population (3.2 compared to a national average of 3.7; OECD, 2011), while North American gardeners tended to come from household sizes 


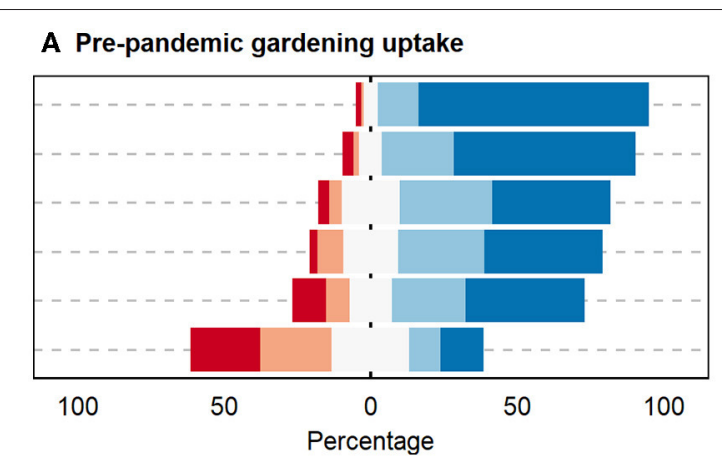

Response

Totally agree

Agree

Neither agree nor disagree

B Gardening uptake during the pandemic

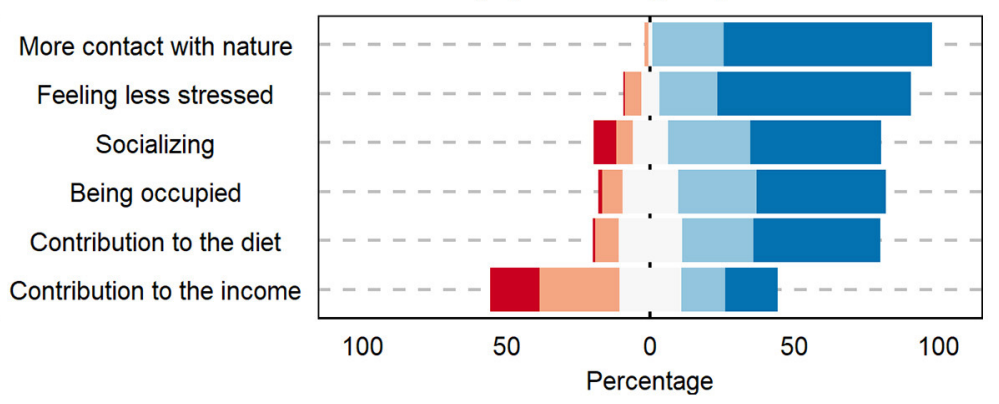

Percentage

FIGURE 6 | Respondents' perceptions of the benefits provided by urban gardening, differentiated according to the time of their gardening uptake. There were no significant differences in the perception of benefits between gardeners who started pre- or during the pandemic. (A) Pre-pandemic gardening uptake. (B) Gardening uptake during the pandemic.

representative of the overall population (2.5 in Detroit and 2.4 in Phoenix, compared with a national average of 2.57; OECD, 2011; Chenarides et al., 2020).

Younger age groups were over-represented in our sample, especially among those who took up urban gardening during the COVID-19 pandemic. This is likely due to our sampling method, i.e., via social media, that provides an interesting perspective into how young people engaged in this traditionally "older" practice. Indeed, older people tend to be more engaged in home food gardening practices, be it before (Machida, 2019; Corley et al., 2021) or during the COVID-19 pandemic (Chenarides et al., 2020), and may be the most captured demographic in surveys (Marsh et al., 2021). A reason for greater participation of midage people in the present research could be the accessibility to resources. During the several lockdown periods in Santiago, nonessential commerce was closed and people were forced to buy items different than food online. This could have been a stronger limitation for older people, especially those living alone, who could have a lower internet proficiency restricting their access to online shopping options to get seeds, soil, or any other resource.

Gardeners, both those who started pre- and during the pandemic, gained their first experience in this practice mostly in their adulthood and had a regular connection with public greenspaces before the COVID-19 pandemic. Unlike elsewhere, where urban gardening may be part of settled cultural traditions or a frequent means of families' self-sufficiency, in Santiago it is an activity that has become more popular over the last few years (Contesse et al., 2018). Consequently, while other studies identify that childhood experiences relate to the likelihood of practicing home food gardening as an adult, even during the COVID-19 pandemic (Katz, 2020; Mullins et al., 2021; Sunga and Advincula, 2021), most of our respondents experienced home food gardening for the first time during their adulthood. Strong relationships with greenspaces can also be related to nature relatedness such as through nature interactions (Oh et al., 2021) and high interest in gardening (Pérez-Urrestarazu et al., 2021).
We found similar relationships, with a high proportion of gardeners reporting having a close relationship with public greenspaces pre-pandemic, as they declared having visited public greenspaces at least once a week before the pandemic started. Thus, drivers relating to nature-relatedness may be a strong predictor of gardening interest and motivation.

Private greenspaces are the norm in Santiago, where only $13 \%$ of greenspaces are public and $52.6 \%$ consist of households' backyards (Espinoza, 2018), and this was reflected in our respondent pool within which most had private access to a food garden. Additionally, the general amount of green area available—both private and public — can vary from 2.26 to $36.8 \%$ of the area depending on the district and its development level (Espinoza, 2018). Thus, access to greenspaces for gardening, both public and private, is limited to those who can afford to live in districts with higher development levels. Yet typically, most of the gardeners (85\%) lived in districts with high or medium development levels, with more opportunities to access spaces for gardening and reap its benefits (Contesse et al., 2018), then potentially reinforcing inequalities. This was even emphasized in the COVID-19 pandemic context as community gardens were closed, and restricting even more the possibilities for gardening without private access to greenspaces. There was still variation within those that had access to private spaces for gardening, with most of gardeners living in houses as opposed to apartments, similarly to gardeners in several countries of the Global North (Schupp and Sharp, 2012; Vávra et al., 2018). Nevertheless, in a highly urbanized city as Santiago, access to a houseeither rented or purchased-is scarce and overall expensive, though mostly relates to having access to an outdoor garden. This explains why the majority of the respondents declared food gardening in front or backyard gardens. However, the pandemic led apartment-dwellers to take up urban gardening despite space restrictions (Mullins et al., 2021). Similarly, the proportion of gardeners from our study who lived in apartments was slightly, though not significantly, higher within those who 
took up gardening during the pandemic. In Santiago, domestic gardens and balcony sizes are probably under the threshold that makes large size gardening more plausible (Darly et al., 2021), explaining why most respondents described their garden as of either small or medium size. However, this reflects the willingness of gardeners to grow food at home even with spatial limitations.

\section{What Are the Main Perceived Barriers of Home Food Gardening During the COVID-19 Pandemic?}

Gardeners experienced both structural barriers and behavioral barriers to their gardening practices. Structural barriers, related to urban policy and planning (Katz, 2020), are on the top of the challenges people faced when home gardening during the COVID-19 pandemic. Lack of or inadequate space for gardening is the most prevalent barrier among gardeners, and also in other studies, pandemic restrictions often limited access to suitable areas for food gardening such as community gardens, and forcing people to garden in their private space (Katz, 2020; Nicola et al., 2020). Other authors have distinguished spatial concerns as a relevant barrier for gardening both before (Eng et al., 2019) and during the COVID-19 pandemic in which this arose as one of the main reasons for not growing food at home at all (Mullins et al., 2021).

A greater behavioral barrier identified by gardeners in this research was limited knowledge of food gardening, especially for those who took up home food gardening during the COVID-19 pandemic. This points to a lack of formal support from either the government or organizations to educate people producing food at home during the pandemic, and forcing people to learn by themselves. Generally, sharing with other gardeners is how people learn about food gardening (Mullins et al., 2021), while promoting support within the food gardening communities (Kingsley et al., 2020). Nevertheless, the pandemicrelated restrictions made such social interactions difficult because community gardens were closed and social contacts were restricted. Mullins et al. (2021) found that only 13.2\% of new gardeners contacted other gardeners or communities for advice while taking up gardening during the pandemic, while Katz (2020) identified lack of knowledge as one of the main barriers for gardening during the pandemic, especially for new gardeners. However, alternative means of gaining knowledge such as online platforms facilitated access to gardening knowledge and support during this period (Katz, 2020; Montefrio, 2020; Mullins et al., 2021; Sunga and Advincula, 2021).

Lack of time to garden was the second most cited barrier for gardeners, despite some pre-pandemic gardeners highlighting how the pandemic allowed them to spend more time on home gardening. Other studies agree that during the COVID-19 pandemic, people spent more time in home food gardening than before the pandemic, mainly because of the social distancing restrictions (Lades et al., 2020; Pérez-Urrestarazu et al., 2021; Sunga and Advincula, 2021). In our study, we can assume that some people lacked time because they continued to work in a different place or, if they stayed at home, they had other time-consuming tasks such as online working, childcare and homeschooling or other hobbies (Katz, 2020).

Finally, reduced access to physical resources (e.g., water, seeds, and soil) was a critical barrier, also recognized by other recent research. Resources for gardening were difficult to get from the beginning of the pandemic: they were rapidly sold out, prices increased and stocks were affected by interruptions in the provisioning chain (Katz, 2020; Nicola et al., 2020; RiveraFerre et al., 2021). This, along with the mostly inexistent support from the government or organizations to provide or facilitate resources, limited the possibilities of developing and maintaining food gardens.

\section{What Are the Main Perceived Benefits of Home Food Gardening During the COVID-19 Pandemic?}

Home food gardening during the COVID-19 pandemic provided an alternative contact with nature, which could help to compensate for the restrictions of visiting public greenspaces. While gardening ornamental plants at home encourages contact with nature, previous studies have recognized that growing one's food creates a much closer bond (Ambrose et al., 2020; Sunga and Advincula, 2021). Planting the seeds, monitoring their growth, watering them daily, harvesting the vegetables, and eating them, makes home gardening a meaningful practice, especially in pandemic times in which being in contact with nature has been mostly restricted to the private sphere (Ambrose et al., 2020; Sunga and Advincula, 2021). Respondents of this research expressed their relationship with nature through growing food at home, unveiling how home food gardening created awareness of the overall relevance of ecosystems and nature.

Strongly related to the restorative capacity of nature (Scott et al., 2020; Soga et al., 2021), our results emphasize the relationship between home food gardening and improved mental health. This confirms recent studies showing how gardening was important for nature connection and individual stress release during the pandemic (Egerer et al., 2022), and how home gardening specifically is effective in improving people's mental and emotional health during the stressful pandemic context (Sunga and Advincula, 2021). Indeed, home food gardening was reported as the third activity with positive impact on emotional well-being during the COVID-19 pandemic in Ireland (Lades et al., 2020), and is amongst the top 15 for affect, happiness and meaningfulness in the USA (Ambrose et al., 2020). We thus contribute to confirm the positive impact of home food gardening for mental health in different geographical and cultural settings.

As in previous crises in history, the present research supports the role of home food gardening to cope with food insecurity issues. Santiago was strongly affected by the side effects of the COVID-19 pandemic. In the first months, people started panic shopping because of food shortages and generalized loss of employment made it difficult to support their families (Cerda, 2020). Although the present study did not explore the type and amount of food production, it gives an idea of how most gardeners perceived their food production to significantly 
contribute to their diet, by helping them reduce shopping trips and finding vegetables otherwise not available when stores were closed. This is consistent with recent studies that have explored the contribution of home gardening to nutritional security (Lal, 2020), general physical health, and food security in the pandemic context (Mullins et al., 2021).

The contribution of home food gardening to physical health was also due to the physical activity that this practice entails. During the COVID-19 pandemic, there was a substantive reduction of it during confinement and lockdowns measurements (Montefrio, 2020). That is why some authors highlight the value of gardening, since maintaining a garden usually involves some degree of physical exercise while cleaning weeds, planting, and harvesting (Katz, 2020; Corley et al., 2021). In our study, most gardeners stated that growing food at home allowed them to stay occupied. Nevertheless, this represented not only more physical activity (Machida, 2019; Sofo and Sofo, 2020) but also was a meaningful and motivating hobby in uncertain times (Katz, 2020) or had important educational value, e.g., when carried out with children.

The restrictions derived from the COVID-19 pandemic imposed limits for person-to-person social interactions in an unprecedented way. According to the present research, home food gardening allowed people to socialize during a time in which social contact was highly restricted, thus revealing how growing food at home can act as a social activity, benefiting people in coping with social isolation and loneliness (Machida, 2019; Kasar and Karaman, 2021; Mullins et al., 2021). Also, in normal times, home food gardening brought communities together, strengthened social networks, and increased social capital (Gallaher et al., 2013; Machida, 2019). Recent studies have also identified these benefits during the COVID-19 pandemic, be it by being a social productive behavior in the Philippines (Sunga and Advincula, 2021), by pointing out the active desire of food gardeners to interact and connect with other gardeners to share and support each other regarding producing food at home (Mullins et al., 2021), by reaching their neighbors and the community for resources and advice (Katz, 2020), or by bringing families together, especially while involving children in the gardening processes (Chenarides et al., 2020).

At the same time, significant vegetable production can reduce the amount of money necessary for food groceries, while overproduction of vegetables or seed production can be sold for extra income. Consequently, home food gardens are known to contribute to income generation at the household level, with benefits varying between geographic context (Galhena et al., 2013). Such benefit was not perceived by most of our respondents, consistently with recent studies during the COVID19 pandemic, which found that families did not have significant savings through gardening (Mullins et al., 2021). This could be explained by their recent uptake in gardening: gardeners may not have had enough time to learn how to obtain high food production in such a short time, considering that some crops need several months to be productive. In addition, financial and knowledge investments to start the practice may have occurred. In all, we can potentially assume that such a mixed perception could change as gardeners gain experience in growing more productive crops on a long run. Improving incomes through home food gardening could also be enhanced by the development of facilitating platforms to sell extra vegetable produced to others within the nearby community.

\section{How Willing Are Gardeners During the COVID-19 Pandemic to Continue Practicing It in the Future?}

Despite the many challenges people faced while gardening during the 1st year of the COVID-19 pandemic, its benefits were more meaningful, leading to nearly all gardeners to express a desire to continue the practice in the future. However, willingness to continue gardening is not guaranteed, especially for those who have recently started gardening. Cases following previous crises are varied. After some historical crises such as World War I, when the need to grow own food as a subsistence strategy decreased, some people continued practicing it but more as a recreational or leisure activity (Schupp and Sharp, 2012) yet declines in home gardening could also be observed since people returned to their "normal" lives (Herrmann, 2015). Consequently, understanding to which point the COVID-19 pandemic was able to motivate a whole new generation of home food gardeners with long-term participation and permanent engagement with gardening will be critical.

\section{Limitations}

Although we surveyed many people in the study area, our research has some limitations. First, we cannot compare our findings to pre-pandemic behavior and perception of the people interviewed as the study was initialized during the pandemic, relating the crisis moment to home gardening as the study focus per se. However, it would be beneficial to follow the trend of increased home food gardening in the future to see if people continue with the practice. Second, the recruitment design targeted people that had access to online resources, including Instagram. This might have biased our sample toward younger people, and could have excluded other members of society such as elderly or people with less interest in internetrelated activities. However, our sample size still represented an important share of the population, as two third of the Chilean population regularly uses Instagram (Statista Research Department, 2021). Additionally, our specific recruitment via social media also brought new insights as opposed to studies that assessed older generations primarily. To add some proportion of respondents without access to internet (for example with personal assistance) would have been helpful to make this sample more representative for the overall population of the study area. That is, our database could be widened if people were also interviewed face-to-face. Unfortunately, this was not possible due to the pandemic situation that included severe lockdowns in the study region-a matter of fact that we targeted in our study specifically-but should be taken into account in possible future studies outside the pandemic context. Third, in our sample, many people were living in districts of high and medium development, as opposed to Santiago's general population, which includes about half of its population 
in districts with low development. Therefore, the share of respondents with access to home gardens might have been over-represented, and future studies should specifically target low development districts. However, this might also be because lack of access to gardens in districts of low development levels hindered people from carrying out urban gardening. Finally, as we targeted active gardeners in our study, only people with some (even small) opportunity to garden were included, and we saw many alternative ways of active gardening also without access to private land.

\section{CONCLUSION}

Many home gardeners here expressed their intention to continue gardening, posing a relevant point to reflect on how this practice can be facilitated and managed in the future. Our insights in the socio-demographic backgrounds of gardeners, and that these mattered only in some few points for different perceptions in the gardening benefits and barriers, suggest that large parts of society may be supported by such practices in difficult times. However, in Santiago, food gardening initiatives are not wellintegrated in urban policy (Contesse et al., 2018). Our findings into the spatial context of home food gardens (often private settings in and around houses) also shows how injustice in access to green areas in cities may manifest in crisis times. This highlights for the specific context of a metropolitan region in South America that access to gardens and green space are a relevant topic if such practices are promoted, to contribute to a just society and support healthy life in cities. This insight opens the door to reflect on how to provide areas for growing food in highly urbanized contexts where public and private greenspaces may be unequally distributed. In Santiago, where access to soil can be extremely scarce, providing more private greenspaces is difficult to achieve. Instead, public or shared spaces within communities and neighborhoods can be an option to make this practice more accessible to all residents. Thus, transforming existing underused spaces such as sidewalks, roof tops or community areas can be a suitable alternative that has been already explored in previous research (Sofo and Sofo, 2020), but not specifically promoted in Santiago. Likewise, the practice of home food gardening can be a relevant tool to cope with the adverse consequences of the pandemic by informing public

\section{REFERENCES}

Ambrose, G., Dasb, K., Fandb, Y., and Ramaswamia, F. (2020). Is gardening associated with greater happiness of urban residents? A multiactivity, dynamic assessment in the Twin-Cities region, USA. Landscape Urban Plan. 198:103776. doi: 10.1016/j.landurbplan.2020. 103776

Buckner, J. D., Abarno, C. N., Lewis, E. M., Zvolensky, M. J., and Garey, L. (2021). Increases in distress during stay-at-home mandates during the COVID-19 pandemic: a longitudinal study. Psychiatr. Res. 298:113821. doi: $10.1016 /$ j.psychres.2021.113821

Casanova, P. (2016). Catastro de huertos urbanos de Santiago: aproximación a su estado actual y su contribución a la soberanía alimentaria. (Master Thesis), Universidad Alberto Hurtado, Santiago, Chile. initiatives to promote healthier lifestyles during this and future crises contexts, and beyond.

\section{DATA AVAILABILITY STATEMENT}

The raw data supporting the conclusions of this article will be made available by the authors, without undue reservation.

\section{ETHICS STATEMENT}

Ethical review and approval was not required for the study on human participants in accordance with the local legislation and institutional requirements. Written informed consent for participation was not required for this study in accordance with the national legislation and the institutional requirements.

\section{AUTHOR CONTRIBUTIONS}

CC and LF: substantial contributions to the conception of the work. CC, LF, and ME: contributions to the design of the work. CC and SG: substantial contributions to the acquisition, analysis, or interpretation of data for the work. CC: drafting the work. LF, ME, and SG: revising the work critically. All authors contributed to the article and approved the submitted version.

\section{FUNDING}

This publication was supported by the German Research Foundation (DFG) within the funding programme Open Access Publishing.

\section{ACKNOWLEDGMENTS}

We want to thank all the enthusiastic gardeners that have participated in our study.

\section{SUPPLEMENTARY MATERIAL}

The Supplementary Material for this article can be found online at: https://www.frontiersin.org/articles/10.3389/fsufs. 2022.841386/full\#supplementary-material

Cepić, S., and Tomićević-Dubljević, J. (2017). Urban community and allotment gardens: research trends and a look ahead. Agricult. Forestr. 63, 191-200. doi: 10.17707/AgricultForest.63.4.20

Cerda, C. (2020). Urban Gardening as a Response to Food Supply Issues in Dense Urban Areas During the COVID-19 Crisis. The Nature of Cities. Available online at: https://www.thenatureofcities.com/2020/09/30/urban-gardeningas-a-response-to-food-supply-issues-in-dense-urban-areas-during-thecovid-19-crisis/ (accessed August 16, 2021).

Chenarides, L., Grebitus, C., Lusk, J., and Printezis, I. (2020). Who practices urban agriculture? An empirical analysis of participation before and during the COVID-19 pandemic. Agribusiness 37, 142-159. doi: 10.1002/agr. 21675

Cheval, B., Sivaramakrishnan, H., Maltagliati, S., Fessler, L., Forestier, C., Sarrazin, P., et al. (2020). Relationships between changes in self-reported 
physical activity and sedentary behaviours and health during the coronavirus (COVID-19) pandemic in France and Switzerland. J. Sports Sci. 39, 699-704. doi: 10.1080/02640414.2020.1841396

Contesse, M., van Veliet, B., and Lenhart, J. (2018). Is urban agriculture urban green space? A comparison of policy arrangements for urban green space and urban agriculture in Santiago de Chile. Land Use Pol. 71, 566-577. doi: 10.1016/j.landusepol.2017.11.006

Corley, J., Okely, J., Taylor, A., Page, D., Welstead, M., Skarabela, B., et al. (2021). Home garden use during COVID-19: associations with physical and mental wellbeing in older adults. J. Environ. Psychol. 73:101545. doi: 10.1016/j.jenvp.2020.101545

Darly, S., Feuillet, T., and Laforêt, C. (2021). Home gardening and the social divide of suburban space: methodological proposal for the spatial analysis of a social practice in the greater Paris Urban Area. Sustainability 13:3243. doi: 10.3390/su13063243

Egerer, M., Lin, B., Kingsley, J., Marsh, P., Diekmann, L., and Ossola, A. (2022). Gardening can relieve human stress and boost nature connection during the COVID-19 pandemic. Urban Forestr. Urban Green. 68:127483. doi: 10.1016/j.ufug.2022.127483

Eng, S., Khun, T., Jower, S., and Murro, M. J. (2019). Healthy lifestyle through home gardening: the art of sharing. Nutr. Rev. 13, 347-350. doi: $10.1177 / 1559827619842068$

Espinoza, S. (2018). Estudio revela que la mayoría de las áreas verdes en las ciudades chilenas son privadas. El Mercurio. Available online at: https://estudio \negsurbanos.uc.cl/estudio-ocuc-revela-que-la-mayoria-delas-areas-verdes-en-las-ciudades-chilenas-son-privadas/ (accessed September 20, 2021).

Ferreira, A. J. D., Marquez, R., Santos, C., and Martins, M. (2018). Urban agriculture: a tool for towards more resilient urban communities? Environ. Sci. Health 5, 93-97. doi: 10.1016/j.coesh.2018.06.004

Gajardo, S. (2019). Region Metropolitana de Santiago: Indice de prioridad social de comunas 2019. Seremi de desarrollo social y familia metropolitana. Santiago: Julio.

Galhena, D. H., Freed, R., and Maredia, K. M. (2013). Home gardens: a promising approach to enhance household food security and wellbeing. Agri. Food Secur. 2:8. doi: 10.1186/2048-7010-2-8

Gallaher, C. M., Kerr, J. M., Njenga, M., Karanja, N. K., and WinklerPrins, A. M. G. A. (2013). Urban agriculture, social capital, and food security in the Kibera slums of Nairobi, Kenya. Agric Hum Values. 30, 389-404. doi: 10.1007/s10460-013-9425-y

Garre-Olmo, J., Turró-Garriga, O., Martí-Lluch, R., Zacarías-Pons, L., AlvesCabratosa, L., Serrano-Sarbosa, D., et al. (2021). Changes in lifestyle resulting from confinement due to COVID-19 and depressive symptomatology: a cross-sectional a population-based study. Comprehens. Psychiatr. 104:152214. doi: 10.1016/j.comppsych.2020.152214

Herrera, M. S. (2020). Huertos escolares en establecimientos educacionales municipales de Santiago de chile: biodiversidad de plantas e invertebrados, entorno y tipos de manejo. (Master Thesis), Pontificia Universidad Católica de Chile Facultad de agronomía e ingeniería forestal Dirección de investigación y postgrado Magíster en recursos naturales, Santiago, Chile.

Herrmann, M. M. (2015). The modern day "victory garden". Proc. Eng. 118, 647-653. doi: 10.1016/j.proeng,.2015.08.498

INE (2017). Censos de Población y Vivienda. Instituto Nacional de Estadísticas. Available online at: https://www.ine.cl/estadisticas/sociales/censos-depoblacion-y-vivienda (accessed November 15, 2021).

Inostroza, L., Baur, R., and Csaplovics, E. (2013). Urban sprawl and fragmentation in Latin America: a dynamic quantification and characterization of spatial patterns. J. Environ. Manag. 115, 87-97. doi: 10.1016/j.jenvman.2012.11.007

Kasar, K. S., and Karaman, S. (2021). Life in lockdown: social isolation, loneliness and quality of life in the elderly during the COVID-19 pandemic: a scoping review. Geriatr. Nurs. 45:1222-1229. doi: 10.1016/j.gerinurse.2021.03.010

Katz, H. (2020). Crisis Gardening: Addressing Barriers to Home Gardening During the COVID-19 Pandemic. (Tesis). Hamilton College, Environmental Studies, Clinton, NY, United States.

Kingsley, J., Foenander, E., and Bailey, A. (2020). "It's about community": exploring social capital in community gardens across Melbourne, Australia. Urban Forestr. Urban Green. 49:126640. doi: 10.1016/j.ufug.2020.126640
Lades, L. K., Laffan, K., Daly, M., and Delaney, L. (2020). Daily emotional wellbeing during the COVID-19 pandemic. Br. J. Health Psychol. 25, 902-911. doi: 10.1111/bjhp. 12450

Lal, R. (2020). Home gardening and urban agriculture for advancing food and nutritional security in response to the COVID-19 pandemic. Food Secur. 12, 871-876. doi: 10.1007/s12571-020-01058-3

Ma, N. L., Peng, W., Soon, C. F., Noor Hassim, M. F., Misbah, S., Rahmatz, Z., et al. (2021). Covid-19 pandemic in the lens of food safety and security. Environ. Res. 193:110405. doi: 10.1016/j.envres.2020.110405

Machida, D. (2019). Relationship between community or home gardening and health of the elderly: a web-based cross-sectional survey in Japan. Environ. Res. Public Health 16:138. doi: 10.3390/ijerph160 81389

Marsh, P., Diekmann, L. O., Egerer, M., Lin, B., Ossola, A., and Kingsley, J. (2021). Where birds felt louder: the garden as a refuge during COVID-19. Wellbeing Sp. Soc. 2:100055. doi: 10.1016/j.wss.2021. 100055

Montefrio, M. J. (2020). Interrogating the "productive" home gardener in a time of pandemic lockdown in the Philippines. Food Foodways 28, 216-225. doi: 10.1080/07409710.2020.1790142

Mullins, L., Charlebois, S., Finch, E., and Music, J. (2021). Home food gardening in Canada in response to the COVID-19 pandemic. Sustainability 13:63056. doi: 10.3390/su13063056

Nicola, S., Ferrante, A., Cocetta, G., Bulgari, R., Nicoletto, C., Sambo, P., et al. (2020). Food supply and urban gardening in the time of COVID-19. Bullet. UASVM Horticult. 77:51. doi: 10.15835/buasvmcn-hort:2020.0051

OECD (2011). Doing Better for Families. Available online at: https://www.oecd.org/social/soc/doingbetterforfamilies.htm (accessed October 3, 2021).

Oh, R. Y. R., Fielding, K. S., Nghiem, T. P. L., Chang, C.-C., Shanahan, D. F., Gaston, K. J., et al. (2021). Factors influencing nature interactions vary between cities and types of nature interactions. People Nat. 3, 405-417. doi: 10.1002/pan3.10181

Pérez-Urrestarazu, L., Kaltsidi, M. P., Nektarios, P. A., Markakis, G., Loges, V., Perini, K., et al. (2021). Particularities of having plants at home during the confinement due to the COVID-19 pandemic. Urban Forestr. Urban Green. 59:126919. doi: 10.1016/j.ufug.2020.126919

Pouso, S., Borja, A., Fleming, L. E., Gómez-Baggethun, E., White, M. P., and Uyarra, M. C. (2021). Contact with blue-green spaces during the COVID-19 pandemic lockdown beneficial for mental health. Sci. Tot. Environ. 756:143984. doi: 10.1016/j.scitotenv.2020.143984

Rivera-Ferre, M. G., López-i-Gelats, F., Ravera, F., Oteros-Rozas, E., di Masso, M., Binimelis, R., et al. (2021). The relation of food systems with the COVID-19 pandemic: causes and consequences. Agricult. Syst. 191:103134. doi: 10.1016/j.agsy.2021.103134

Sandorf, A. (2020). Coronavirus: Half of the Humanity Now on Lockdown as 90 Countries Call for Confinement. Available online at: https://www.euronews. com/2020/04/02/coronavirus-in- europe-spain-s-death-toll-hits-10-000after-record-950-new-deaths-in-24-hou (accessed August 15, 2021).

Schupp, J. L., and Sharp, J. S. (2012). Exploring the social bases of home gardening. Agricult. Hum. Values 29, 93-105. doi: 10.1007/s10460-0119321-2

Scott, T. L., Masser, B. M., and Pachana, N. A. (2020). Positive aging benefits of home and community gardening activities: older adults report enhanced selfesteem, productive endeavours, social engagement and exercise. SAGE Open Medi. 8, 1-13. doi: 10.1177/2050312120901732

Shimpo, N., Wesener, A., and McWilliam, W. (2019). How community gardens may contribute to community resilience following an earthquake. Urban Forestr. Urban Green. 38, 124-132. doi: 10.1016/j.ufug.2018. 12.002

Sofo, A., and Sofo, A. (2020). Converting home spaces into food gardens at the time of COVID-19 quarantine: all the benefits of plants in this difficult and unprecedented period. Hum. Ecol. 48, 131-139. doi: 10.1007/s10745-020-00147-3

Soga, M., Evans, M. J., Tsuchiya, K., and Fukano, Y. (2021). A room with a green view: the importance of nearby nature for mental health during the COVID-19 pandemic. Ecol. Appl. 31:eap.2248. doi: 10.1002/eap.2248 
Statista Research Department (2021). Social Media Usage in Chile-Statistics and Facts. Available online at: https://www.statista.com/topics/6985/social-mediausage-in-chile/ (accessed January 1, 2022).

Sunga, A. B., and Advincula, J. L. (2021). The "plantito/plantita" home gardening during the pandemic. Commun. Psychol. Glob. Perspect. 7, 88-105. doi: 10.1285/I24212113V7I1P88

United Nations (2020). Senior Officials Sound Alarm Over Food Insecurity, Warning of Potentially "Biblical" Famine, in Briefings to Security Council Title. Available online at: https://www.un.org/press/en/2020/sc14164.doc.html (accessed June 8, 2021).

Vávra, J., Megyesi, B., Duzí, B., Craig, T., Klufová, R., Lapka, M., and Cudínová, E. (2018). Food self-provisioning in Europe: an exploration of sociodemographic factors in five regions. Rural Sociol. 83, 431-461. doi: 10.1111/ruso. 12180

World Health Organization (2020). Director-General's Opening Remarks at the Media Briefing on COVID-19. Available online at: https://www.who. int/director-general/speeches/detail $/ \$+\$$ who-director-general-s-openingremarks-at-the-media-briefing-on-covid-19 (accessed March 11, 2020).
Conflict of Interest: The authors declare that the research was conducted in the absence of any commercial or financial relationships that could be construed as a potential conflict of interest.

Publisher's Note: All claims expressed in this article are solely those of the authors and do not necessarily represent those of their affiliated organizations, or those of the publisher, the editors and the reviewers. Any product that may be evaluated in this article, or claim that may be made by its manufacturer, is not guaranteed or endorsed by the publisher.

Copyright (c) 2022 Cerda, Guenat, Egerer and Fischer. This is an open-access article distributed under the terms of the Creative Commons Attribution License (CC BY). The use, distribution or reproduction in other forums is permitted, provided the original author(s) and the copyright owner(s) are credited and that the original publication in this journal is cited, in accordance with accepted academic practice. No use, distribution or reproduction is permitted which does not comply with these terms. 